\title{
Low-loss Shielded Through-Silicon Vias Filled With Multi-Walled Carbon
}

\section{Nanotube Bundle}

Jinrong Su, Runbo Ma, Xinwei Chen, Liping Han, Rongcao Yang, and Wenmei Zhang*

College of Physics and Electronics, Shanxi University, Shanxi, 030006, People's Republic of China (e-mail: zhangwm@sxu.edu.cn).

Abstract - In this paper, the multi-walled carbon nanotube bundle (MWCNTB) based shielded through-silicon via (S-TSV) is proposed and the compact expression for the equivalent conductivity of MWCNTB ( $\sigma_{\mathrm{MWCNTB}}$ ) is deduced to calculate the resistance of MWCNTB based S-TSV (MS-TSV). Then, the electrical characteristics including the $S$ parameters, attenuation constant and time delay are investigated. The results indicate that $\left|S_{21}\right|$ of MS-TSV increases with the increase of the outermost diameter of MWCNT and decrease of the thickness of the shielding layer. Compared with the copper filled S-TSV (CuS-TSV), the MS-TSV has a larger $\left|S_{21}\right|$, smaller attenuation and shorter time delay. Finally, the impact of the geometrical parameters on the conductivity of MS-TSV is analyzed. Also, the minimum packing density of MWCNTB satisfying $\sigma_{\text {MWCNTB }} \geq \sigma_{\mathrm{Cu}}$ has been deduced. The results show that the outermost diameter of MWCNT has the most significant impact on the conductivity of MS-TSV, and thicker MWCNT is helpful to increase the conductivity of MS-TSV, decrease the packing density of MWCNTB and reduce manufacturing difficulty.

Index terms-Forward transmission coefficient, multi-walled carbon nanotube (MWCNT), propagation constant, through-silicon via (TSV), time delay

\section{Introduction}

Through-silicon vias (TSVs) providing a vertical connection between chips is the primary technology for three-dimensional (3-D) integrated circuits (ICs) to realize high-performance, high-functionality, and high-density. However, the silicon substrate noise will degrade the performance of the system when a high frequency signal is transmitted vertically through the TSV. To improve the performance, the coaxial through-silicon via (C-TSV) has been proposed [1]. Much research on the electrical model and characterization of C-TSV has been conducted. For example, in [2], the closed-form formulas for the per-unit-length resistance and inductance of C-TSVs are derived from the theory of quasi-magnetostatic fields. In [3], frequency- and temperature-dependent model and characteristic of circular and square C-TSVs are investigated. In [4], the C-TSV is fabricated and its $S$ parameters are examined and compared with signal-ground paired TSVs. These studies indicate that C-TSV can serve as a low-noise and low-crosstalk structure.

Note that the conductor materials of C-TSV in the above reports are the copper, tungsten and 
aluminum. The skin effect and electromigration of these metals will degrade the performance and reliability of ICs for high- frequency, high-temperature applications [5]. Fortunately, the emerging carbon nanotube (CNT) have a long mean free path (MFP), high thermal conductivity, and high current-carrying capability such that it is the most promising material to replace copper for very large scale integration interconnects in the future [6]-[8]. Also, lots of researches on the CNT based TSV have been done. For example, in [9], a submicron single-walled CNT (SWCNT) based TSV was characterized and the energy delay product of the SWCNT based TSV was investigated. In [10], the accurate impedance model of SWCNT and multi-walled CNT (MWCNT) based TSV was proposed. Literatures [11] and [12] presented the wideband model of SWCNT/MWCNT based differential TSV and mixed CNT based differential TSV, respectively. The results indicate that CNT based TSVs can provide superior performance than copper TSV. However, there is no report on CNT based C-TSV.

In this paper, the MWCNT bundle is used as the conductor material of shielded TSV (S-TSV, since the MWCNT is also coaxial, "shielded" is used to avoid confusion). The expression of the MFP is simplified using nonlinear curve-fitting method. Based on it a compact expression for the conductivity of MWCNTB ( $\left.\sigma_{\text {MWCNTB }}\right)$ is deduced and is used to calculate the resistance of MWCNTB based S-TSV (MS-TSV). Then, the electrical characteristics of MS-TSV are calculated and compared with that of CuS-TSV (copper based S-TSV). Finally, parametric study on the conductivity of MWCNTB is discussed. Also, the minimum packing density of MWCNTB satisfying $\sigma_{\mathrm{MWCNTB}} \geq \sigma_{\mathrm{Cu}}$ is deduced.

This paper is organized as follows. In Section II, the expression for the conductivity of MWCNT is deduced and the resistance is calculated. Then, the $S$ parameters, attenuation constant and time delay of MS-TSV are calculated in Section III. Impact of parameters on conductivity of MWCNTB, and the minimum packing density of MWCNTB are analyzed in Section IV. Finally, a conclusion is drawn in Section V.

\section{Equivalent electrical model of MS-TSV}

Fig. 1(a) shows the schematic of MS-TSV. It is composed of the inner via, insulation layer and outer shielding layer. For simplicity, the substrate and the silicon dioxide layer between the shielding layer and the substrate are omitted. The inner via and the outer shielding layer are filled with MWCNT bundle and the insulation layer is filled with benzocyclobutene (BCB). $r_{1}, r_{2}$ and $r_{3}$ are the radii of the inner via, insulation layer and shielding layer, respectively. $t_{\text {ins }}$ and $t_{s}$ are the thickness of the insulation layer and shielding layer, respectively. $H_{\mathrm{tsv}}$ is the height of the MS-TSV and $D_{\text {out }}$ is the outermost 
diameter of the MWCNT.

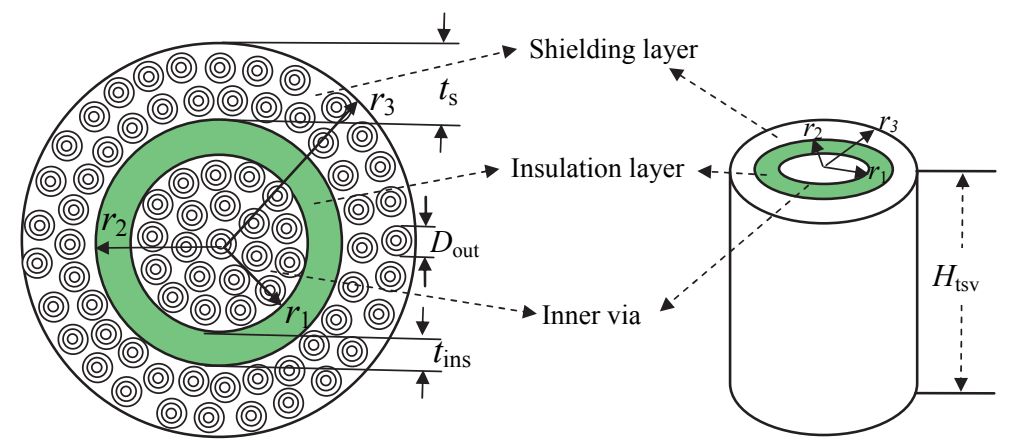

(a)

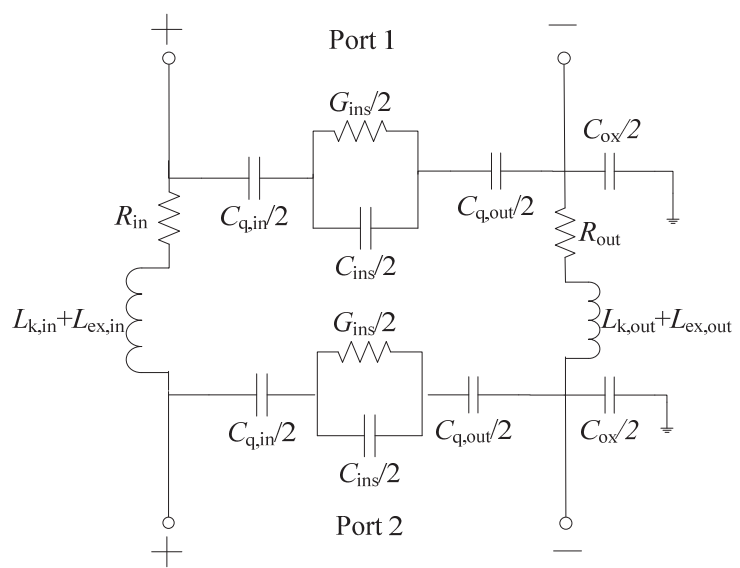

(b)

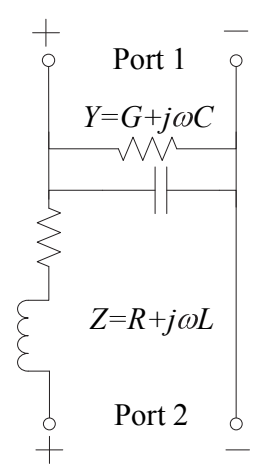

(c)

Fig. 1 (a) Schematic (b) equivalent circuit and (c) equivalent transmission line model of MS-TSV

Fig. 1(b) is the equivalent circuit model of the MS-TSV in Fig. 1(a). In this figure, the subscripts in and out represent the inner via and outer shielding layer, respectively. $L_{\mathrm{k}}$ and $C_{q}$ are the kinetic inductance and quantum capacitance of MWCNTB. $C_{\mathrm{q}, \text { in }}, C_{\mathrm{q}, \text { out }}, L_{\mathrm{k}, \text { in }}$ and $L_{\mathrm{k}, \text { out }}$ can be calculated by (10) - (14) given in [13]. Here, the electrostatic capacitance $C_{\mathrm{E}}$ between neighboring shells is ignored because it is seven orders of magnitude larger than $C_{\mathrm{q}}$. The tunneling conductance of MWCNT can also be ignored according to the measured result in [14]. $L_{\mathrm{ex}}, C_{\mathrm{ins}}$ and $G_{\mathrm{ins}}$ are the external inductance, capacitance and conductance of the insulation layer, and can be calculated using the formulas for the coaxial transmission line given in [15]. $C_{\mathrm{ox}}$ is the capacitance between the shielding layer and the substrate, and it has no impact on the performance of S-TSV. $R_{\text {in }}$ and $R_{\text {out }}$ are the per-unit-length resistance of the inner via and outer shielding layer, respectively, and can be calculated by

$$
\begin{gathered}
R_{\mathrm{in}}(T)=\frac{1}{\pi r_{1}^{2} \sigma_{\mathrm{MWCNTB}}(T)} \\
R_{\text {out }}(T)=\frac{1}{\pi\left(r_{3}^{2}-r_{2}^{2}\right) \sigma_{\mathrm{MWCNTB}}(T)}
\end{gathered}
$$

where $\sigma_{\mathrm{MWCNTB}}(T)$ is the temperature-related effective conductivity of MWCNT bundle, and can be 
expressed as

$$
\sigma_{\mathrm{MWCNTB}}(T)=H_{\mathrm{tsv}} \cdot \frac{2}{\sqrt{3}}\left(D_{\mathrm{out}}+s\right)^{-2} \sum_{\text {shell }} R_{\mathrm{M}, i}^{-1}(T)
$$

where $D_{\text {out }}$ is the diameter of the outermost shell of a MWCNT. For densely packing MWCNT bundle, $s$ $\approx 0.34 \mathrm{~nm}$ is the van der Waals gap between the neighboring CNTs. $R_{\mathrm{M}, i}(T)$ is the self impedance of the $i^{\text {th }}$ shell (from the outer to the inner) of an individual MWCNT and can be expressed as [13]

$$
R_{\mathrm{M}, i}(T)=\frac{h}{2 q^{2} N_{i}(T)}\left(1+\frac{H_{\mathrm{tsv}}}{\lambda_{i}(T)}\right)
$$

where $h$ is Planck's constant and $q$ is the quantity of electric charge. $N_{i}(T)$ is the number of conducting channels of the $i^{\text {th }}$ shell,

$$
N_{i}(T)=\left\{\begin{array}{l}
a T D_{i}+b, \quad D_{i}>d_{\mathrm{T}} / T \\
2 / 3, \quad D_{i}<d_{\mathrm{T}} / T
\end{array}\right.
$$

where $a=3.87 \times 10^{-4} \mathrm{~nm}^{-1} \mathrm{~K}^{-1}, b=0.2$ and $d_{\mathrm{T}}=1300 \mathrm{nmK}[16] . D_{i}=D_{\text {out }}-2 \mathrm{~s}(i-1)$, is the diameter of the $i^{\text {th }}$ shell and $\lambda_{i}(T)$ is the temperature-dependent electron MFP of the $i^{\text {th }}$ shell. Using nonlinear curve-fitting method, we simplify the formulas (10)-(17) for MFP in [17] as

$$
\lambda_{i}(T)=\left(\frac{k_{1}}{D_{i}}+\frac{1}{k_{2} H_{t s v}}\right)^{-1}
$$

where $k_{1}=10^{-5}(0.4 T-43), k_{2}=6.7 \times U$ and $U$ is the bias voltage of MS-TSV.

The interval between two adjacent shells is the van der Waals gap $s \approx 0.34 \mathrm{~nm}$. Note that $s$ is small such that the difference of $R_{\mathrm{M}, i}(T)$ between two adjacent shells is small too. Therefore, the summation in (3) can be approximated by an integral. Assuming $D_{\text {out }}=2 D_{\text {in }}$ (similar to the experimental measurements in [18]), we have

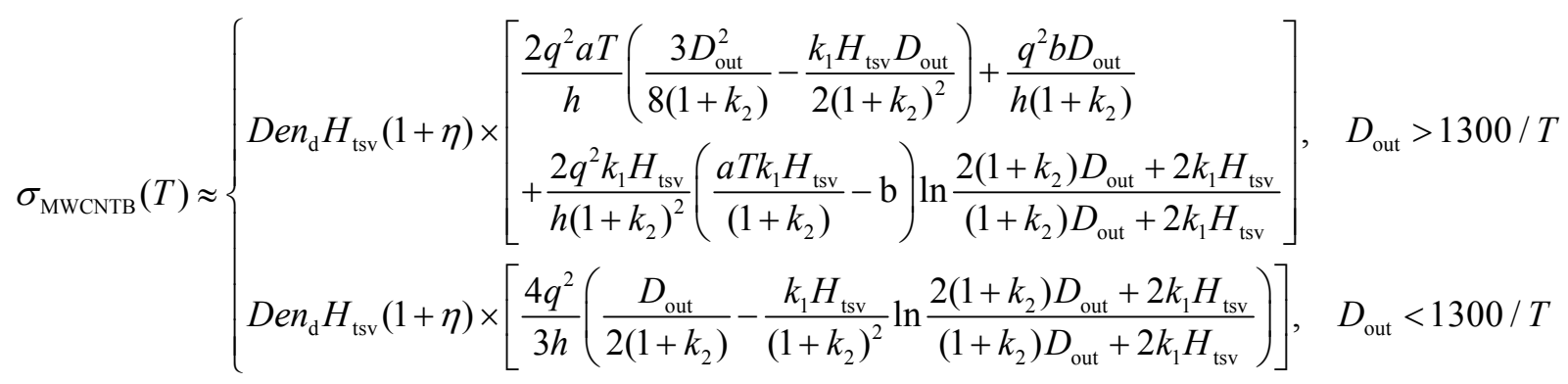

where $\eta=0.55+0.2 e^{-0.06 H_{\mathrm{tsv}}}$ is correction factor, and $D e n_{\mathrm{d}}$ is the total number of CNTs per unit area, and can be expressed as

$$
\operatorname{Den}_{\mathrm{d}} \approx \frac{2}{\sqrt{3}\left(D_{\text {out }}+\mathrm{s}\right)^{2}}
$$


Note that $D e n_{\mathrm{d}}$ can also be defined as the density of densely packing bundle.

In order to verify the accuracy of (7), the calculated results using (3) and (7) are compared as shown in Fig. 2. It depicts that the two results agree well. The average (maximum) percentage error is $2.96 \%$ $(5.53 \%), 0.94 \%(3.82 \%)$ and $0.95 \%(3.28 \%)$ when $H_{\mathrm{tsv}}$ is $30 \mu \mathrm{m}, 50 \mu \mathrm{m}$ and $80 \mu \mathrm{m}$, respectively. Hence, (7) can be used to analyze $\sigma_{\mathrm{MWCNTB}}(T)$.

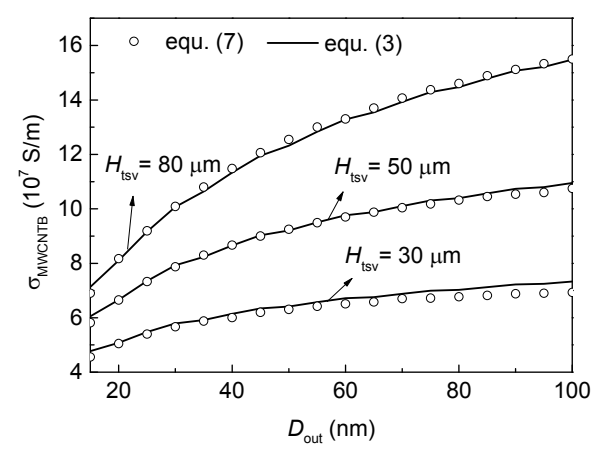

Fig. $2 \sigma_{\text {MWCNTB }}$ calculated using (3) and (7)

The equivalent circuit in Fig. 1(b) can be further reduced to the transmission line model shown in Fig. 1(c). The per-unit-length admittance and impedance are,

$$
\begin{gathered}
Y=G+j \omega C=\left[\left(G_{\mathrm{ins}}+j \omega C_{\mathrm{ins}}\right)^{-1}+1 / j \omega C_{\mathrm{q}, \text { in }}+1 / j \omega C_{\mathrm{q}, \text { out }}\right]^{-1} \\
Z=R+j \omega L=R_{\mathrm{in}}+R_{\mathrm{out}}+j \omega\left(L_{\mathrm{k}, \mathrm{in}}+L_{\mathrm{k}, \mathrm{out}}+L_{\mathrm{ex}}\right)
\end{gathered}
$$

\section{The electrical characteristics of MS-TSV}

The propagation constant $\gamma$ and the characteristic impedance in the transmission line model of MS-TSV can be expressed as,

$$
\begin{gathered}
\gamma=\alpha+j \beta=\sqrt{(R+j \omega L)(G+j \omega C)} \\
Z_{\mathrm{c}}=\sqrt{(R+j \omega L) /(G+j \omega C)}
\end{gathered}
$$

The ABCD matrix and $S$ parameters can be calculated by [15]

$$
\begin{aligned}
{\left[\begin{array}{ll}
A & B \\
C & D
\end{array}\right]=} & {\left[\begin{array}{ll}
\cosh \left(\gamma H_{\mathrm{tsv}}\right) & \mathrm{Z}_{\mathrm{c}} \sinh \left(\gamma H_{\mathrm{tsv}}\right) \\
\sinh \left(\gamma H_{\mathrm{tsv}}\right) / \mathrm{Z}_{\mathrm{c}} & \cosh \left(\gamma H_{\mathrm{tsv}}\right)
\end{array}\right] } \\
& \left\{\begin{array}{l}
S_{11}=\frac{A+B / Z_{0}-C Z_{0}-D}{A+B / Z_{0}+C Z_{0}+D} \\
S_{21}=\frac{2}{A+B / Z_{0}+C Z_{0}+D}
\end{array}\right.
\end{aligned}
$$

where $Z_{0}=50 \Omega$. Using the formulas for $R, L, G$ and $C$ addressed in Section II, the $S$ parameters and $\alpha$ can be calculated and the results are shown in Figs. 3-4. For comparison, some results of CuS-TSV are also provided. The baseline dimensions of S-TSV are $D_{\text {out }}=20 \mathrm{~nm}, H_{\mathrm{tsv}}=30 \mu \mathrm{m}, t_{\mathrm{ins}}=2 \mu \mathrm{m}, r_{1}=5 \mu \mathrm{m}$, $r_{2}=7 \mu \mathrm{m}, r_{3}=8 \mu \mathrm{m}$ and $U=0.2 \mathrm{~V}$. The conductivity of $\mathrm{Cu}$ at temperature $T$ is 
$5.8 \times 10^{7} \times[1+0.00385(T-300)] \mathrm{S} / \mathrm{m}$ and the complex permittivity of BCB filled in the insulation layer is $2.6 \varepsilon_{0}(1-\mathrm{j} 0.0008)$.

\subsection{S parameters}

Fig. 3 plots the $\left|\mathrm{S}_{21}\right|$ of MS-TSV for different $t_{\text {ins. }}$ In Fig. 3(a), $\left|\mathrm{S}_{21}\right|$ of MS-TSV increases with $t_{\text {ins. At }}$ $100 \mathrm{GHz}$, as $t_{\text {ins }}$ increases from $1 \mu \mathrm{m}$ to $3 \mu \mathrm{m},\left|\mathrm{S}_{21}\right|$ increases from $-0.068 \mathrm{~dB}$ to $-0.025 \mathrm{~dB}$ and the

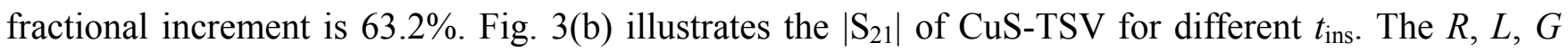
and $C$ of CuS-TSV are calculated according to the formulas for the coaxial transmission line in [15]. The varying of $\left|\mathrm{S}_{21}\right|$ with $t_{\text {ins }}$ is similar to that of MS-TSV. However, the fractional increment of $\left|\mathrm{S}_{21}\right|$ for $\mathrm{Cu}-\mathrm{TSV}$ is $85.7 \%$, which is larger than that of MS-TSV. This implies that $t_{\text {ins }}$ has greater impact on $\left|\mathrm{S}_{21}\right|$ of CuS-TSV than that of MS-TSV. This is because $\left|\mathrm{S}_{21}\right|$ increases with the decrease of $C$. For CuS-TSV,

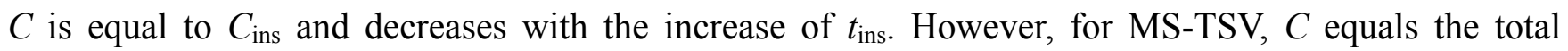
capacitance of $C_{\mathrm{q}, \text { in }}, C_{\mathrm{q}, \text { out }}$ and $C_{\mathrm{ins}}$ in series. As $t_{\mathrm{ins}}$ increases, $C_{\mathrm{q}, \text { out }}$ increases and $C_{\mathrm{ins}}$ decreases. The two opposite effects partially counteract that the impact of $t_{\text {ins }}$ on $C$ is weakened. Moreover, the $\left|S_{21}\right|$ of MS-TSV is larger than that of CuS-TSV because $C$ and $G$ of MS-TSV are less than that of CuS-TSV.

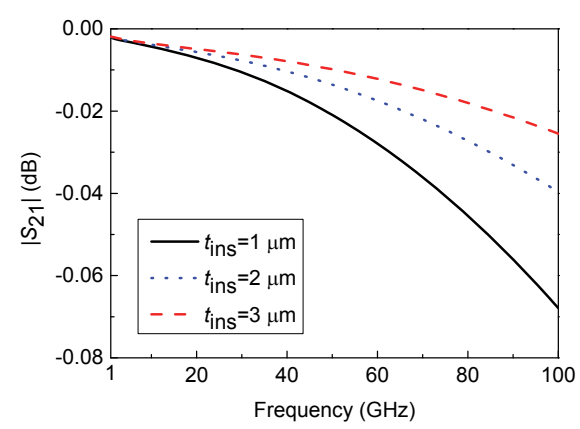

(a)

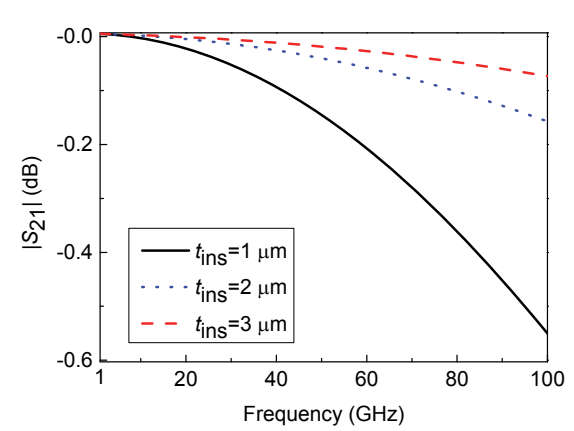

(b)

Fig. $3\left|S_{21}\right|$ varying with frequency for different $t_{\text {ins }}$ : (a) MS-TSV, (b) CuS-TSV

Fig. 4 displays the effect of other parameters. Fig. 4(a) depicts $\left|S_{21}\right|$ for different $t_{\mathrm{s}}$. For comparison, the results of CuS-TSV are also provided. For MS-TSV, $\left|\mathrm{S}_{21}\right|$ decreases with $t_{\mathrm{s}}$. This is because the total number of individual tubes in a MWCNT bundle $\left(N_{\text {tot }}\right)$ increases with $t_{\mathrm{s}}$. Then, $C_{\mathrm{q}, \text { out }}$ increases simultaneously. As a result, the signal leakage through the shielding layer increases. However, for CuS-TSV, $t_{\mathrm{s}}$ doesn't affect the impedance of the shielding layer due to the skin effect. Also, $C$ and $G$ of CuS-TSV don't vary with $t_{\mathrm{s}}$. Thus, $\left|\mathrm{S}_{21}\right|$ doesn't vary with $t_{\mathrm{s}}$. This implies that for CuS-TSV, one can adjust $t_{\mathrm{s}}$ according to the manufacturing difficulty and cost, and don't have to worry about the transmission performance. However, for MS-TSV the shielding layer should be as thinner as possible to decrease loss. 


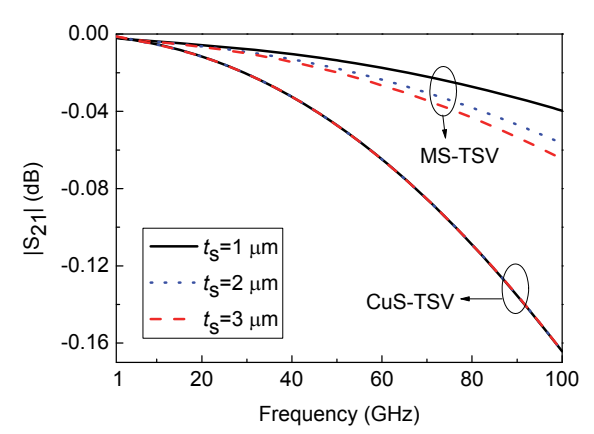

(a)

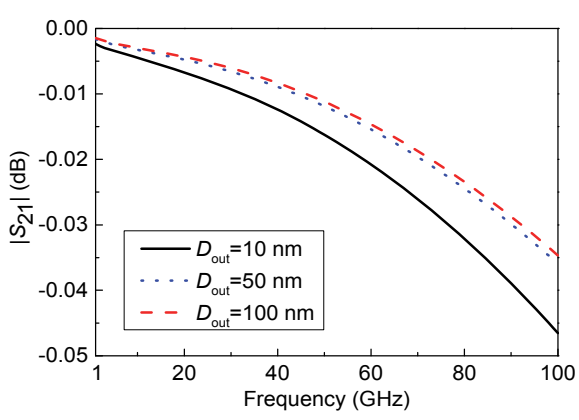

(b)

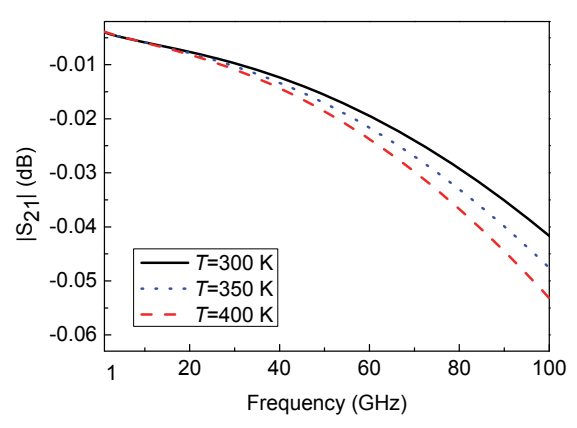

(c)

Fig. $4\left|S_{21}\right|$ varying with frequency for different (a) $t_{\mathrm{s}}$, (b) $D_{\text {out }}$, and (c) $T$

Fig. 4(b) plots $\left|\mathrm{S}_{21}\right|$ of MS-TSV for different $D_{\text {out }}$ One can see that $\left|\mathrm{S}_{21}\right|$ increases with $D_{\text {out. This }}$ is because the resistance of MWCNTB decreases with the increase of $D_{\text {out }}$ Then, the conductor loss is reduced simultaneously. In addition, it can be observed that the impact of $D_{\text {out }}$ on $\left|\mathrm{S}_{21}\right|$ weakens as $D_{\text {out }}$ increases. For example, when $D_{\text {out }}$ varies from $10 \mathrm{~nm}$ to $50 \mathrm{~nm}$ and $100 \mathrm{~nm},\left|S_{21}\right|$ at $20 \mathrm{GHz}$ increases from $-0.0067 \mathrm{~dB}$ to $-0.0048 \mathrm{~dB}$ and $-0.0044 \mathrm{~dB}$, and the increments are $0.0019 \mathrm{~dB}$ and $0.0004 \mathrm{~dB}$, respectively. This is because a larger $D_{\text {out }}$ will produce more conducting channels for every individual MWCNT. However, $N_{\text {tot }}$ will decrease simultaneously. For example, $N_{\text {tot }}$ of the bundle with a radius of 5 $\mu \mathrm{m}$ are about $8.5 \times 10^{5}$ and $9 \times 10^{3}$ for $D_{\text {out }}=10 \mathrm{~nm}$ and $D_{\text {out }}=100 \mathrm{~nm}$, respectively.

Fig. 4(c) illustrates $\left|\mathrm{S}_{21}\right|$ of MS-TSV for different $T$. It states that $\left|\mathrm{S}_{21}\right|$ decreases slightly as $T$ rises. This is because as $T$ increases, $\lambda_{i}(T)$ decreases and $N_{i}(T)$ increases. Then, $R_{\text {in }}$ and $R_{\text {out }}$ increase with the decrease of $\lambda_{i}(T)$, and $C_{\mathrm{q}}$ increases with the increase of $N_{i}(T)$. As a result, the conductor loss and the loss cause by leakage increase.

\subsection{The attenuation constant}

The attenuation constant $\alpha$ is the real part of $\gamma$ and can be calculated using (11). Fig. 5 plots $\alpha$ of MS-TSV and CuS-TSV. One can observe that the attenuation when $t_{\text {ins }}=1 \mu \mathrm{m}$ is larger than that of 2 $\mu \mathrm{m}$ for both MS- and CuS-TSV. This is because $C_{\text {ins }}$ increases with the decrease of $t_{\text {ins. }}$ Additionally, one can see that $\alpha$ of MS-TSV is dramatically less than that of CuS-TSV. To investigate the reason for that, we derive the expression of $\alpha$ from (11) as

$$
\alpha=\frac{\sqrt{2 \omega C}}{2}\left(\sqrt{\omega^{2} L^{2}+R^{2}}-\omega L\right)^{1 / 2}
$$

Note that $G$ is ignored since it is far smaller than $j \omega C$. In (15), L of MS- and CuS-TSV are mainly determined by $L_{\mathrm{ex}}$, and they are close. While, MS-TSV has a less $C$ (as addressed previously) and R (because MWCNT can suppress the skin effect significantly) than that of CuS-TSV. As a result, $\alpha$ of MS-TSV is less than that of CuS-TSV. 


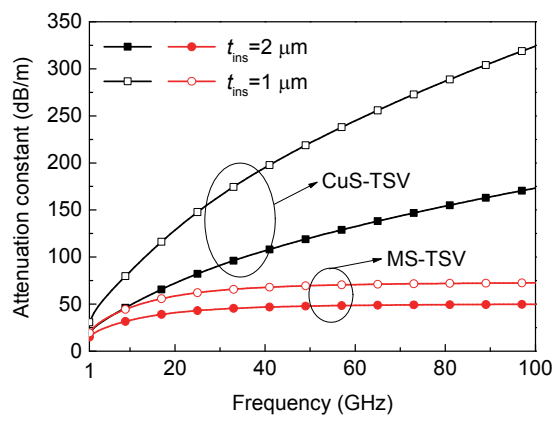

Fig. $5 \alpha$ of MS- and CuS-TSV

\subsection{Time delay}

The time delay of S-TSV $t_{d}$ can be calculated by [19]

$$
t_{d}=\sqrt{R C} / \omega
$$

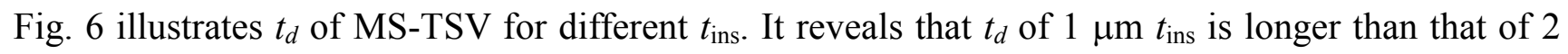
$\mu \mathrm{m}$ since $C$ increases as $t_{\text {ins }}$ decreases. Additionally, the results of CuS-TSV are also plotted here for comparison. One can observe that $t_{d}$ of MS-TSV is a little smaller than that of CuS-TSV. The reason for this is similar as that for $\alpha$.

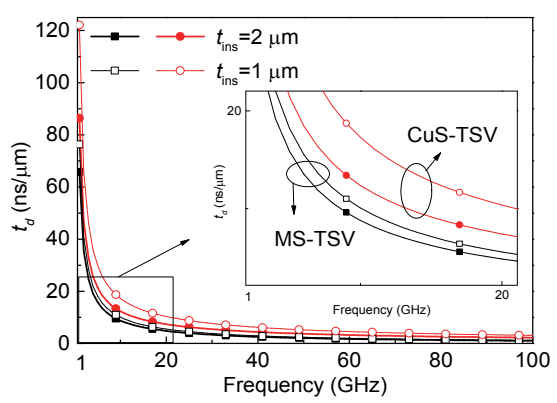

Fig. $6 t_{d}$ of MS-TSV and CuS-TSV

\section{Parametric study on the conductivity and packing density of MWCNTB}

\subsection{Parametric study on $\sigma_{M W C N T B}(T)$}

To investigate the impact of the variables affecting $\sigma_{\mathrm{MWCNTB}}$ in (7), we define the sensitivity coefficient $\sigma_{x}$ as

$$
\sigma_{x}=\frac{\partial \sigma_{\mathrm{MWCNTB}}}{\partial x}
$$

where $x$ represents the variables $H_{\mathrm{tsv}}, D_{\text {out }}, T$ and $U$. Table I lists $\sigma_{x}$ when $D_{\text {out }}=20 \mathrm{~nm}, H_{\mathrm{tsv}}=30 \mu \mathrm{m}$, $T=300 \mathrm{~K}$ and $U=0.2 \mathrm{~V}$. It states that $D_{\text {out }}$ has the largest impact and $T$ has the weakest one on $\sigma_{\text {MwCNTB. }}$ Increasing $D_{\text {out }}$ is an effective method to increase $\sigma_{\text {MWCNTB }}$. In addition, $\sigma_{U}<0$ indicates that $\sigma_{\text {MWCNTB }}$ decreases with the increase of $U$.

\begin{tabular}{ccccc}
\multicolumn{5}{c}{ Table I SENSITIVITY COEFFICIENTS OF $\sigma_{\text {MWCNTB }}(T)$} \\
\hline Variables & $H_{\text {tsv }}$ & $D_{\text {out }}$ & $T$ & $U$ \\
\hline$\sigma_{x}$ & $1.14\left(\mathrm{~S} / \mu \mathrm{m}^{2}\right)$ & $784\left(\mathrm{~S} / \mu \mathrm{m}^{2}\right)$ & $0.047(\mathrm{~S} / \mu \mathrm{m} \cdot \mathrm{K})$ & $-69.1(\mathrm{~S} / \mu \mathrm{m} \cdot \mathrm{V})$ \\
\hline
\end{tabular}




\subsection{The packing density of $M W C N B$}

The packing density affects $\sigma_{\mathrm{MWCNTB}}(T)$ directly, and will affect the loss and attenuation of MS-TSV consequently. In the previous analysis, the MWCNTB is assumed to be densely packed. However, densely packing is difficult in the actual process [20]. Therefore, it is necessary to explore an appropriate packing density satisfying $\sigma_{\mathrm{MWCNTB}} \geq \sigma_{\mathrm{Cu}}$. To simply the expression of $\sigma_{\mathrm{MWCNTB}}$, we substitute the expression in the square brackets of (7) with $R_{\mathrm{M} \text {,int }}(T)$, and get,

$$
\operatorname{DenH}_{\mathrm{tsv}}(1+\eta) R_{\mathrm{M}, \mathrm{int}}(T) \geq \sigma_{\mathrm{Cu}}(T)
$$

Then, Den can be derived from (18) as

$$
\operatorname{Den} \geq \frac{\sigma_{\mathrm{Cu}}(T)}{H_{\mathrm{tsv}} R_{\mathrm{M}, \mathrm{int}}(T)(1+\eta)}
$$

Denoting the minimum Den satisfying (19) as $D e n_{\min }$, we define the sparseness degree $D_{\text {spa }}$ as

$$
D_{\text {spa }}=\frac{D e n_{\text {min }}}{D e n_{\mathrm{d}}}=\frac{D e n_{\text {min }} \sqrt{3}\left(D_{\text {out }}+s\right)^{2}}{2}
$$

where $D e n_{\mathrm{d}}$ is the density of densely packing. If $D e n_{\mathrm{d}}$ is $100 \%, D_{\text {spa }}$ means the minimum percentage satisfying $\sigma_{\mathrm{MWCNTB}} \geq \sigma_{\mathrm{Cu}}$.

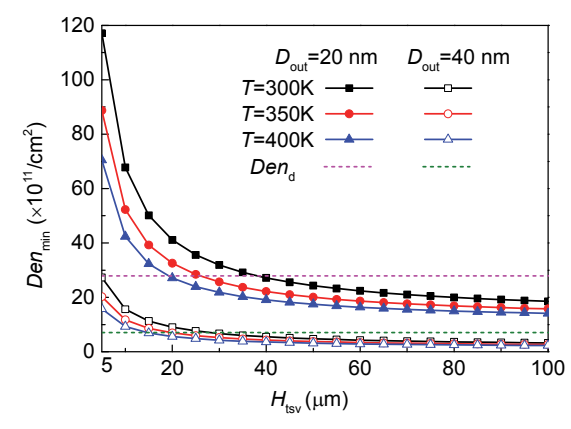

(a)

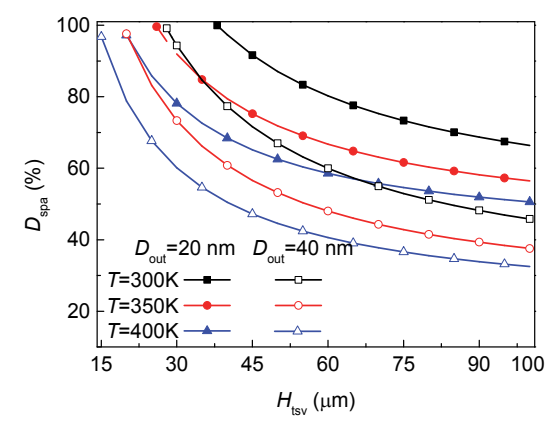

(b)

Fig. 7 (a) $D e n_{\min }$ and (b) $D_{\text {spa }}$ varying with $H_{\text {tsv }}$ at different $T$.

To find the MS-TSV satisfying $\sigma_{\mathrm{MWCNTB}} \geq \sigma_{\mathrm{Cu}}, D e n_{\min }$ and $D_{\mathrm{spa}}$ varying with $D_{\text {out }}$ and $H_{\mathrm{tsv}}$ at different temperatures are studied and the results are shown in Fig. 7. In Fig. 7(a), the two dash lines are $D n_{d}=$ $2.75 \times 10^{11} \mathrm{~cm}^{-2}$ and $7.09 \times 10^{10} \mathrm{~cm}^{-2}$ for $D_{\text {out }}=20 \mathrm{~nm}$ and $40 \mathrm{~nm}$, respectively. The MWCNTB can't be realized if $D e n_{\min }>D e n_{\mathrm{d}}$. In addition, one can see that $D e n_{\min }$ declines with the increase of $D_{\mathrm{out}}, H_{\mathrm{tsv}}$ and $T$. For example, when $H_{\mathrm{tsv}}=40 \mu \mathrm{m}$ and $D_{\text {out }}=20 \mathrm{~nm}$, as $T$ rises from $300 \mathrm{~K}$ to $400 \mathrm{~K}$, $D e n_{\text {min }}$ decreases from $2.72 \times 10^{11} \mathrm{~cm}^{-2}$ to $1.91 \times 10^{11} \mathrm{~cm}^{-2}$. Besides, when $D_{\text {out }}=20 \mathrm{~nm}$ at $300 \mathrm{~K}$, as $H_{\text {tsv }}$ increases from $40 \mu \mathrm{m}$ to $60 \mu \mathrm{m}, D_{e n}$ min decreases from $2.72 \times 10^{11} \mathrm{~cm}^{-2}$ to $2.24 \times 10^{11} \mathrm{~cm}^{-2}$. Fig. $7(\mathrm{~b})$ reveals that $D_{\text {spa }}$ declines as $H_{\text {tsv }}$ increases. For example, when $T=300 \mathrm{~K}, D_{\text {out }}=20 \mathrm{~nm}, D_{\text {spa }}$ are $97.31 \%$ and $66.3 \%$ for $H_{\mathrm{tsv}}=40 \mu \mathrm{m}$, and $100 \mu \mathrm{m}$, respectively. In addition, $D_{\text {spa }}$ decreases with the increase of $D_{\text {out. }}$ For 
example, when $T=300 \mathrm{~K}, H_{\mathrm{tsv}}=100 \mu \mathrm{m}, D_{\text {spa }}$ are $66.38 \%$ and $45.81 \%$ for $D_{\text {out }}=20 \mathrm{~nm}$ and $40 \mathrm{~nm}$, respectively. Besides, both $D e n_{\min }$ and $D_{\text {spa }}$ decrease as $T$ rises because $\sigma_{\mathrm{Cu}}$ decreases with $T$.

Above all, thicker MWCNT should be used for shorter vias to satisfy $\sigma_{\mathrm{MWCNTB}} \geq \sigma_{\mathrm{Cu}}$ easily, and the manufacturing difficulty can be reduced simultaneously.

\section{Potential fabrication method}

A potential fabrication process for MS-TSV is suggested in Fig. 8. It start with the deep reactive ion etching (DRIE) an annular trench in substrate 1 [21]. Then, a layer of $\mathrm{SiO}_{2}$ is sputtered inside the trench for insulation. Next, MWCNT forests are grown by thermal chemical vapor deposition (CVD) to fill the trench. Again, DRIE is utilized to remove the silicon post inside the annular trench. At the same time, MWCNT forests are grown on substrate 2 and then transferred to the via in substrate 1. Furthermore, the BCB layer is then spin coated to fill the gap between the MWCNT and the sidewall of the via [22]. Finally, the chemical-mechanical polish is used to remove the excess surface materials.

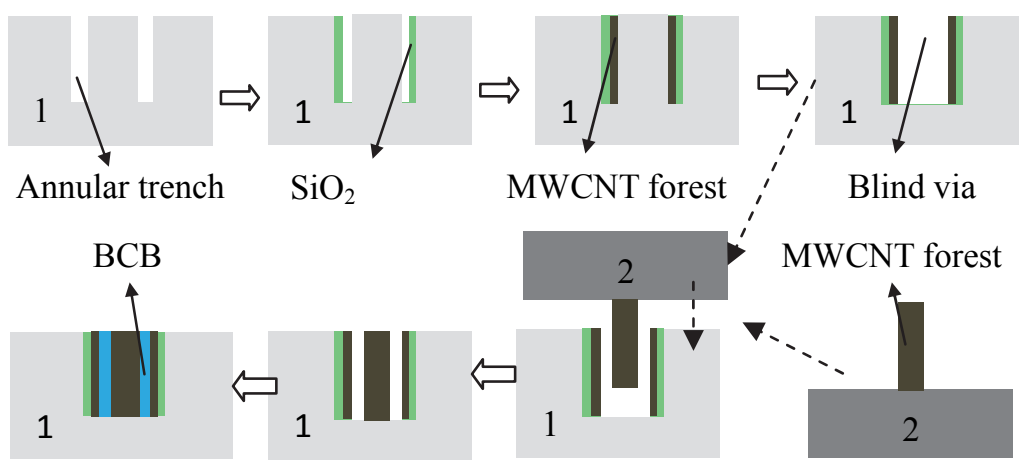

Fig. 8 Illustration of the fabrication process of TSVs.

\section{Conclusions}

High-performance shielded TSV filled with MWCNTB has been proposed in this paper. A compact expression for the conductivity of MWCNTB has been deduced and the resistance of MS-TSV is calculated. Then, together with formulas of $L, G$ and $C$, the $S$ parameters, the attenuation constant and time delay have been calculated. For comparison, the results of CuS-TSV have also been provided. It indicates that MS-TSV has larger $\left|S_{21}\right|$, smaller attenuation and shorter time delay than CuS-TSV. In addition, $\left|S_{21}\right|$ of MS-TSV increases with the increase of $D_{\text {out }}$ and decrease of $t_{\mathrm{s}}$. Also, the parametric study on $\sigma_{\mathrm{MWCNTB}}$ has been done. The results show that $D_{\text {out }}$ has the largest impact on $\sigma_{\mathrm{MWCNTB}}$. Finally, the minimum packing density satisfying $\sigma_{\mathrm{MWCNTB}} \geq \sigma_{\mathrm{Cu}}$ has been derived. It reveals that thicker MWCNT is helpful to increase the conductivity of MS-TSV and reduce manufacturing difficulty. 


\section{Acknowledgements}

This work was supported by the National Science Foundation of China (61271160), the Research Fund for the Doctoral Program of Higher Education (20121401110009), the Natural Science Young Foundation of Shanxi Province (2014021021-1, 2015011042).

\section{References}

[1] S. W. Ho, V. S. Rao, O. K. N. Khan, S. U. Yoon, V. Kripesh, Development of coaxial shield via in silicon carrier for high frequency application, In: Proc. 8th Electronics Packaging Technology Conference, Dec. 2006, pp. 825-830.

[2] F. Liang, G.F. Wang, D.S. Zhao, and B.Z. Wang, Wideband impedance model for coaxial through-silicon vias in 3-D integration, IEEE Trans. Electron Devices 60(8) ( 2013) 2498-2504.

[3] W.S. Zhao, W.Y. Yin, X.P. Wang, and X.L. Xu, Frequency-and temperature- dependent modeling of coaxial through-silicon vias for 3-D ICs, IEEE Trans. Electron Devices 58(10) (2011) 3358-3368.

[4] S. Adamshick, D. Coolbaugh, M. Liehr, Experimental characterization of coaxial through silicon vias for 3D integration, Microelectronics Journal 46(5) ( 2015) 377-1443.

[5] Y.C. Tan, C.M. Tan, X.W. Zhang, T. C. Chai, and D.Q.Yu, Electromigration performance of through silicon via (TSV) -A modeling approach, Microelectron. Reliab. 50(9-11) (2010) 1336-1340.

[6] H. Li, C. Xu, N. Srivastava and K. Banerjee, Carbon nanomaterials for next-generation interconnects and passives: physics, status, and prospects, IEEE Trans. Electron Devices 56(9) (2009) 1799-1821.

[7] P. G. Collins, M. Hersam, M. Arnold, R. Martel, et al, Current saturation and electrical breakdown in multi-walled carbon nanotubes, Phys. Rev. Lett. 86(14) ( 2001) 3128-3131.

[8] K. M. Liew, C. H. Wong, X. Q. He, and M. J. Tan, Thermal stability of single and multi-walled carbon nanotubes, Phys. Rev. B, Condens. Matter 71 ( 2005) 075424.

[9] W.S. Zhao, L.L. Sun, W.Y. Yin, Y.X. Guo, Electrothermal modeling and characterization of submicron through-silicon carbon nanotube bundle vias for three-dimensional ICs, Micro \& Nano. Letters 9(2) (2014) 123-126.

[10]C. Xu, H. Li, R. Suaya, and K. Banerjee, Compact AC modeling and performance analysis of through-silicon vias in 3-D ICs, IEEE Trans. Electron Devices 57(12) ( 2010) 3405-3417.

[11]W.S. Zhao, J. Zheng, F. Liang, K.W. Xu, X. Chen, and G.F. Wang, Wideband modeling and characterization of differential through-silicon vias for 3-D ICs, IEEE Trans. Electron Devices 63(3) (2016) 1168-1175.

[12]L.B. Qian, , Y.S. Xia, and G. Shi, Electrical modeling and analysis of a mixed carbon nanotube based differential through silicon via in 3-D integration, IEEE Trans. Nanotechnology 15(2) ( 2016) 155-163.

[13]H. Li, W.Y. Yin, K. Banerjee, and J.F. Mao, Circuit modeling and performance analysis of multi-walled carbon nanotube interconnects, IEEE Trans. Electron Devices 55(6) (2008) 1328-1337.

[14] Y. G. Yoon, P. Delaney, and S. G. Louie, Quantum conductance of multiwall carbon nanotubes, Phys. Rev. B, Condens. Matter 66(7) (2002) 073407.

[15] S. Eamo, J.R. Whinnery, T.V. Duzer, Fields and waves in communication electronics. John Wiley \&Sons, Inc., USA, 1993 3rd ed.

[16]A. Naeemi, J.D. Meindl, Performance modeling for single- and multiwall carbon nanotubes as signal and power interconnects in gigascale systems, IEEE Transactions On Electron Devices 55(10) (2008) 2574-2582. 
[17]F. Liang, G.F. Wang, H. Lin, Modeling of self-heating effects in multi-wall carbon nanotube interconnects, Micro \& Nano Letters 6(1) (2011) 52-54.

[18]H. J. Li, W. G. Lu, J. J. Li, X. D. Bai, and C. Z. Gu, Multichannel ballistic transport in multiwall carbon nanotubes, Phys. Rev. Lett. 95(8) (2005) 086601.

[19]I. Ndip, B. Curran, K. Löbbicke, S. Guttowski, H. Reichl, K.-D. Lang, and H. Henke, High-frequency modeling of TSVs for 3-D chip integration and silicon interposers considering skin-effect, dielectric quasi-TEM and slow-wave modes, IEEE Trans. Compon., Packag. Manuf. Technol. 1(10) (2011) 1627-1640.

[20]C. Brun, C.C. Yap, D.L. Tan, S. Bila, S. Pacchini, D. Baillargeat, and B. K. Tay, Flip chip based on carbon nanotube-carbon nanotube interconnected bumps for high-frequency applications, IEEE Trans. Nanotechnology 12(4) ( 2013) 609-615.

[21] Q.W. Chen, C. Huang, Z.M. Tan, and Z.Y. Wang, Low Capacitance Through-Silicon-Vias With Uniform Benzocyclobutene Insulation Layers, IEEE Transactions On Components, Packaging And Manufacturing Technology, 3(5) (2013) 724-731.

[22]T. Wang, S. Chen, D. Jiang, Y.F. Fu, K. Jeppson, L.L. Ye, and J. Liu, Through-Silicon Vias Filled With Densified and Transferred Carbon Nanotube Forests, IEEE Electron Device Letters, 33(3) (2012) 420-422. 